\title{
Convergent Quantification and Physical Support for Teilhard de Chardin's Philosophy Concerning the Human Species and Evolutionary Consciousness
}

\author{
Brendan Lehman1,2, Michael A. Persinger ${ }^{1,2,3,4^{*}}$ \\ ${ }^{1}$ Behavioural Neuroscience, Laurentian University, Sudbury, Canada \\ ${ }^{2}$ Department of Biology, Laurentian University, Sudbury, Canada \\ ${ }^{3}$ Biomolecular Sciences, Laurentian University, Sudbury, Canada \\ ${ }^{4}$ Human Studies Programs, Laurentian University, Sudbury, Canada \\ Email: ${ }^{*}$ mpersinger@laurentian.ca, bx lehman@laurentian.ca
}

Received 27 June 2015; accepted 31 July 2015; published 4 August 2015

Copyright (C) 2015 by authors and Scientific Research Publishing Inc.

This work is licensed under the Creative Commons Attribution International License (CC BY). http://creativecommons.org/licenses/by/4.0/

(c) (i) Open Access

\section{Abstract}

Teilhard de Chardin's integration of geobiological phenomena with philosophical and spiritual perspectives resulted in ideas, such as potentially quantifiable "spiritual energy", the emergence of a "sphere of human thinking" (noosphere) as the next evolutionary stage of the biosphere, and the ultimate expansion of consciousness into the galaxy. Transformations of his concepts into contemporary values that effectively define cosmology, quantum biology, and human cerebral parameters could support his interpretations. Scaled quantification of basic universal energies match the magnitudes measured within the human cerebrum during thinking. Superposition of the magnetic fields associated with thinking upon the intergalactic strength fields for induced changes in magnetic moments of elementary electrons solves for durations that approach the age of the universe or "immortality". The immersion of the human species within both the earth's magnetic field and the Schumann Resonances creates the conditions for producing the "noosphere" and for its potential expansion into space. The rapid development of new computer-based technologies that expose the human population to homogeneous energetic patterns and produce cognitive states consistent with "unifying" the noosphere could be sufficient to produce physical changes which would support Teilhard de Chardin's hypotheses.

\section{Keywords}

Teilhard de Chardin, Spiritual Energy, Quantum Energy, Human Brain, Schumann Resonances,

\footnotetext{
${ }^{*}$ Corresponding author.
}

How to cite this paper: Lehman, B. and Persinger, M. A. (2015). Convergent Quantification and Physical Support for Teilhard de Chardin's Philosophy Concerning the Human Species and Evolutionary Consciousness. Open Journal of Philosophy, 5, 338-350. http://dx.doi.org/10.4236/ojpp.2015.56042 
Immortality, Sphere of Human Thinking (Noosphere), Expansion of Consciousness, Biophysical Evidence, Physical Philosophy, Digital Games, Digital Culture

\section{Introduction}

The “dangerous thoughts" of combining traditional religious and philosophical presumptions with human evolution were articulated during the year 1938 in the manuscript entitled Le Phenomene Humain (The Phenomenon of Man) by Pierre Teilhard de Chardin (1959). He was an original thinker who was attempting to integrate the most fundamental philosophical presumptions of Christian Theology with the scientific theory of evolution. Although his nomenclature reflected this time, the prescience of his concepts was extraordinary and anticipated the central thrust of contemporary neuroscience in general and neurotheology and neurophilosophy in particular. His postulations that evolution is an ascent towards complexity and consciousness that can expand into space and time have significant ramifications for the future of the human species. The emergence of a sphere of human thought, the noosphere, as the third progression after the geosphere of inanimate matter and the biosphere of animate (biological) matter, could manifest unique and different properties. The transformation of his ideas into technologies could contribute to this progression. Like the biosphere affecting the geosphere, the noosphere could affect both.

Merz (1965) discussed the similarities of scientific and religious/philosophical perspectives from the late $19^{\text {th }}$ century with regards to "universal” concepts such as energy. Recently Nordlund (2015) applied modern concepts of physics to St. Augustine and Chung (2015) has integrated aspects of quantum physics with Confucian philosophy. We have assumed that, at their essence, the fundamental operations for the substrates mediating the relationships between the words that thinkers have employed to describe concepts for the boundaries of space and time and those moderating relationships between physical units share a common source of variance. Here we apply quantification from contemporary biophysics, the environmental sciences, and quantum concepts (Persinger et al., 2008) to Teilhard de Chardin's fundamental assumptions that attempt to "bind” biology, cosmology, and the essential metaphysics. The latter has been central to all sciences and philosophies. We then describe an emerging technology whose physical properties could lead to the condition that could precipitate the "global consciousness" and expansion of beyond the terrestrial boundaries that he suggested and described in the 1955 publication of his treatise.

\section{2. "Spiritual" Energy as "Quantum" Level Energy}

Teilhard de Chardin's consternation with the difference between matter and energy and between the physical and the spiritual (p. 67) was similar to many intellects who have grappled with the dichotomous distinctions in general and the mind-body problem in particular. The implicit assumption for this dichotomy is that the differences are qualitative rather than quantitative. His focus was to "connect the two energies of the body and the soul in a coherent manner". He succinctly states "the inner face of the world is manifest deep within our human consciousness and there reflects upon itself...there are dynamic relationships existing between the within and the without of things at a given point in the universe". (Words in italics were indicated in his original work). This perspective that consciousness reflects the universe and it reflects consciousness is remarkably congruent with the phenomenological perspectives of how the human perception (consciousness) affects our measurements of the universe according to Teilhard de Chardin's contemporary and astrophysicist Sir Arthur Eddington (1981).

According to Teilhard de Chardin, "The two energies - of mind and matter-spread respectively through the two layers of the world (the within and the without), have, taken as a whole, much the same demeanor” (p. 69). In contemporary biochemistry and biophysics the two energies are more typically represented as those associated with matter or molecular pathways and those associated with energy or electromagnetic patterns most frequently associated with photons or light. However, one interpretation of his comment is that the two are qualitatively the same but different only quantitatively.

If he were alive today there would very likely be no dilemma. Niels Bohr (1958), one of the primary originators of quantum theory, had stated that the "energy" of thoughts and cognition were likely to be within the mag- 
nitudes of quantum units, very small and very discrete. In other words, the primary difference between energies of the machinery of cells and the machinery of "thought" or "spiritual" patterns is the magnitude. They are not "apples and oranges" but rather different sized apples. The apparent qualitative difference is a perception because the contemporary measurements are not sufficiently precise to discern the quantitative difference. One classic illustrative example is a description from the Ancient Greeks. They assumed that "mind" or "soul" moved as "animal spirits" through tubes and hence was "non-physical". Translated to contemporary language "thoughts" are aggregates of "electromagnetic fields" with measureable intensities that move along axon barrels with discrete lengths. Both are operationally similar and only differ in their quantification.

The matter that occupies the volume of the human brain is organized as cells, primarily neurons and glia cells. The cell is composed of multiple organelles and structures that must be maintained continually in opposition to the forces of "deconstruction". About $10^{-12}$ Joules (the unit of energy, and energy is force over distance) is required per second per cell. The coefficients will vary but the order of magnitude remains more or less similar. This assumes a classic cell with a width of about 10 micrometers.

On the other hand, a single action potential, which is a temporally discrete (about 1 millisecond or $10^{-3} \mathrm{~s}$ ) change in voltage of about $120 \mathrm{mV}\left(1.2 \times 10^{-1} \mathrm{~V}\right)$ acting upon a unit charge (of a proton or an electron) of $1.6 \times$ $10^{-19} \mathrm{~A} \cdot \mathrm{s}$ (Amp second) or a Coulomb, exerts an energy of $2 \times 10^{-20} \mathrm{~J}$ (Persinger, 2010). If there were $10 \mathrm{of}$ these spikes per second or $10 \mathrm{~Hz}$ (the median peak power frequency of the human cerebrum as measured by electroencephalography as "brain waves"), the energy associated with the "electromagnetic" pattern of neurons would be $2 \times 10^{-19} \mathrm{~J}$ per second or about ten million times less than the energy per second required to maintain the metabolic operation of the neuron as cellular matter.

This small amount of energy per action potential, $10^{-20} \mathrm{~J}$, is the same magnitude of quantum that is required to add a base nucleotide to a ribbon of RNA that will ultimately determine the type of protein sequence within the cell. Proteins are constructed of series of amino acids and determine the structures and functions (enzymes) of the cell. In this manner the pattern of action potentials which reflects the impact of the environment upon sensory input is transformed into more or less stable patterns of proteins that are the representations of experience or memory for the individual. This unit of energy is also the same order of magnitude as many of the complex forces, such as the hydrogen bond, that "hold" the chemical units of the biological machinery together (Persinger, 2010).

The range of energies between $10^{-20} \mathrm{~J}$ and $10^{-19} \mathrm{~J}$ is within the band associated with visible light. Since the 1930s photon emissions have been recorded from all living systems (Gurwitch, 1926). The rejuvenation of these measurements, primarily stimulated by Popp (1979), has indicated that the photon emissions are not simply metabolic artifacts but are intimately involved with inter-cell and intra-cell communications (Trushin, 2009; Fels, 2009). Popp (1979) has argued that because all (or simply most) of the energy that has contributed to the evolution of biological systems on this planet originates from the sun, "virtual photons" that reflect this history are represented within the matter of the cell.

Relating consciousness and thinking to photon emissions has been an aspiration of many scientists over the centuries. The concept that "light" is an indicator of properties or processes that reflect positive qualities of space and time are represented in many religious perspectives. If "spiritual” energies are actual energies displayed at very low intensities then biophoton emissions are candidates for the physical equivalence. The measurements require photomultiplier units and are usually within the range of $10^{-12}$ Watts (Joules per second) per meter squared (Dotta et al., 2011). For comparison this irradiant power density (Watts per meter squared) is about 10,000 times weaker than the "dark" on a cloudy, moonless night.

Dotta and his colleagues $(2011,2012)$ measured clear, reversible changes in photon emissions from the right sides of the heads of human participants sitting in hyper-dark chambers when they imagined white light compared to when they did not. The increase in photon emissions from the right side of the head was about $10^{-12} \mathrm{~W}$ per $\mathrm{m}^{2}$. Simultaneous measurement of quantitative shifts in electroencephalographic power indicated strong correlations between the activity within the participants left prefrontal region which is associated with personal intent, decision-making and choice, and the irradiant flux density of the photon emissions from the right side of the head (Dotta et al., 2012).

The importance of this connection between imagination and consciousness and photon emissions from the human brain to Teilhard de Chardin's premises cannot be over-emphasized. These biophotons match his operational description of the essence or evanescent particle (p. 283). They also connect or resolve the dichotomy between matter and energy or mass and spirit in a very quantitative manner (Persinger, 2012a, 2013a). The ve- 
locity of light, even when mildly attenuated in water, is about $2.8 \times 10^{8} \mathrm{~m}$ per second. The width of the plasma cell membrane of a neuron is $10^{-8} \mathrm{~m}$ (10 nanometers). Hence, the time required for a photon to pass through the membrane of a neuron would be about $10^{-16} \mathrm{~s}$. The actual value is overlaps with the time required for an electron in the classical Bohr atom to complete one orbit.

This temporal congruence creates the conditions for any photon originating anywhere in the universe to have sufficient time to transpose the information within the packet into an electron orbit and hence the mass within the membrane of a neuron. It also means that the photons generated within the atoms that compose the cell membrane from the shifts in electron orbits would have the potential to be emitted and traverse anywhere within the universe. What is required is a specific technology or process that facilitates excess correlation between great distances, that is, "non-locality". If the photon is timeless or can exist as virtual patterns within space-time for indefinite periods, then both complexity and connectivity within the entire universe could be accessed potentially. The convergence, the "omega point” was Teilhard de Chardin's description of the maximum level of that complexity and consciousness to which the universe is evolving.

\section{The Personal Universe}

In the chapter concerning the convergence of the person and the omega point, Teilhard de Chardin states that after the catabolism of all of the forms of synthesis (p. 283) and the ultimate dismantlement of biological machinery, primarily only evanescent particles remain. This is remarkably similar to the concept of entropy when the final distributions of energy into the smallest increment of measurement approach equality. He states "Only one reality survives and be capable of succeeding and spanning the infinitesimal and the immense: energy.." There should be a fundamental unit of energy that can expand or is implicitly present through which the expansion can occur.

The concept of the humanized progression of noogenesis (the sphere of thinking) and space-time is similar to the process of a hologram which is defined in many contemporary terms as when the unit reflects the whole and the whole reflects the unit (Rose, 2006). Teilhard de Chardiin expresses this as “...the Universal and Personal... grow in the same direction and culminate simultaneously in each other. (p. 285) and "The conclusion is inevitable that the concentration of a conscious universe would be unthinkable if it did not reassemble in itself all consciousness as well as all the consciousness" (p. 287). The "Omega" concept, in its ultimate principle, can only be a "distinct Centre radiating at the core of a systems of centres; a grouping in which personalization of the All and personalization of the elements reach their maximum, simultaneously, without merging, under the influence of supremely autonomous focus of union” (p. 289).

There are two perspectives that are consistent quantitatively with this concept. If one assumes the individual unit is the human brain and the numbers of neurons within the cerebral cortices is around 25 billion (Pakkenberg \& Gundersen, 1997) but that only about 10 million neurons are required for a concept or a percept and each discharge 10 times per second with an energy of $10^{-20} \mathrm{~J}$, the total energy per second would be $10^{-12} \mathrm{~J}$ per second. Interestingly this is the amount of energy supplied by the "machinery" of the biological systems through glucose metabolism per second for a single neuron, the unit of thinking. That the activity of only one neuron can affect the organization of the activity of the entire cerebral cortices ( $\mathrm{Li}$ et al., 2009) or determine the direction of a complex behavior (Houwelling \& Brecht, 2008) has been shown experimentally.

From Teilhard de Chardin's perspective what is more critical is the energy density of the human cerebrum during thinking. Assuming a typical volume of $\sim 10^{-3} \mathrm{~m}^{3}$ for the cerebrum, the energy density from the $10^{-12} \mathrm{~J}$ (per second) would be about $10^{-9} \mathrm{~J}$ per $\mathrm{m}^{3}$. The value is conspicuous. If the total energy within the universe based upon contemporary estimates of $10^{69} \mathrm{~J}$ (from the mass of $10^{52} \mathrm{~kg}$ ) and the volume of the universe is in the order of $10^{78} \mathrm{~m}^{3}$, the average energy density of the entire set that defines existence is $10^{-9} \mathrm{~J}$ per cubic meter (Persinger, 2013b). This convergence would be a condition expected by the premise that "the whole and the individual reach their maximum without merging and that the universal and the personal grow in the same direction and culminate simultaneously in each other”.

\section{The Uniting Factor}

Any system that is intrinsically connected such that the every element is associated with the whole and the whole is associated with each element, must have some type of operational commonality. For Ernst Mach's concept of the Prominence of the Universe, behavior of any part of the Cosmos is determined by all of its parts 
(Persinger, 2013a). From that perspective any physical field could be conceived as existing anywhere. Teilhard de Chardin's unifying principle is an energy which he defines as love with a rather exotic definition. It "is a more or less direct trace marked on the heart of an element by the psychical convergence of the universe upon itself (p. 291)". Furthermore, "love alone is capable of uniting living beings in such as way as to complete and to fulfill them, for it alone takes them and joins them by what is deepest within themselves". Effectively he states there is some fundamental smallest (deepest) unit within an element through which all elements could interact and could be represented within the entire or maximum set, the universe.

There is evidence for this uniting principle that is the energy found within the essence of human cognition, thinking, and at the most fundamental spatial fabric of the universe: Planck's Length $\left(10^{-35} \mathrm{~m}\right)$. One would expect that equations that are applicable to the subset of the universe should be valid when the whole universe is considered. Force is equal to mass times distance times the square of frequency. For the total mass of $10^{52} \mathrm{~kg}$ over the length of $10^{26} \mathrm{~m}$ multiplied by the intrinsic oscillation of the universe, effectively the square of the reciprocal of Planck's time or the actual Zwitterbewegung of the oscillations (Puthoff, 1989) attributed to oscillations for zero point vacuum oscillations $\left(10^{43} \mathrm{~Hz}\right)$, that is $10^{86} \mathrm{~s}^{-2}$, the total force would be $10^{164} \mathrm{~N}$. To obtain this value one adds the superscripts of the powers of 10 for mass, distance and the squared frequency.

The density of this force, to follow Teilhard de Chardin's direction, would require considering the smallest unit of spatial structure or Planck's Length $\left(10^{-35} \mathrm{~m}\right)$. For simplicity if one assume a Planck's voxel or volume unit of $10^{-105} \mathrm{~m}^{3}$ and the total volume of the universe $\left(10^{78} \mathrm{~m}^{3}\right)$, then there would be $10^{183}$ Planck's voxels within the universe. Hence the force per voxel would be $10^{-19} \mathrm{~N}$. For energy to be realized this force must be applied over a distance. The most predominant distance throughout the universe due primarily to the fact that about $90 \%$ of the composition of the universe is hydrogen (one proton, one electron), is the electromagnetic wavelength associated with the neutral hydrogen line of $21 \mathrm{~cm}\left(2.1 \times 10^{-1} \mathrm{~m}\right)$. The multiplication of this distance by the unit force of $10^{-19} \mathrm{~N}$ is about $10^{-20} \mathrm{~J}$.

This quantum of energy (Persinger, 2010) is the basic unit associated with the action potentials of neurons from which cognition is correlated and from which consciousness is assume to emerge. This unifying factor would presumably exist within the smallest imaginable space, Planck's Length, and the most common element: neutral hydrogen. Such homogeneity of energy could allow the specific form of "connectiveness" that Teilhard de Chardin attributed to "love as an energy" that would be capable of uniting not only all living things but all "things". He states, in prose, the essence of this statement in the section reason for love, as "Expressed in terms of internal energy, the cosmic function of Omega consists in initiating and maintaining within its radius the unanimity of the world's reflective particles” (p. 295).

\section{The Human Brain and the Earth}

The evolutionary continuity of life and consciousness is a central theme within The Phenomenon of Man. Teilhard de Chardin frequently discusses the terrestrial continuity of the evolution of the human "mind" which emerged from the brain. Like many contemporary neuroscientists (Hammeroff \& Penrose, 2014) he linked the degree of complexity with consciousness. What he did not realize because the technology was not present at the time of his insights is that the fundamental characteristics of the volume occupied by the human cerebrum and that of the earth itself are both complex and remarkably similar. Because the earth is $4 \times 10^{7} \mathrm{~m}$ in circumference and the velocity of light is $3 \times 10^{8} \mathrm{~m} \cdot \mathrm{s}^{-1}$ (meters per second) there is a resonance shell between the ionosphere and the earth's surface of about $8 \mathrm{~Hz}$. This has been called the Schumann Resonance (Nickolaenko \& Hayakawa, 2014).

Current estimates of the bulk, coherent velocity of fields of action potentials over relatively large areas of the cerebral cortices are $4.5 \mathrm{~m}$ per s. These recurrent fields display a phase velocity in the order of $25 \mathrm{~ms}$ or " $40 \mathrm{~Hz}$ " and move in a rostral to caudal direction over the surface of cerebrum (Llinas \& Pare, 1991). Assuming the average circumference of a human head is $60 \mathrm{~cm}$, the resonance frequency is about $8 \mathrm{~Hz}$. There are harmonics or higher frequencies which show peaks of power along the frequency continuum separated by about $6 \mathrm{~Hz}$ which are found in the human cerebral power spectra and the Schumann domain at $14 \mathrm{~Hz}, 20 \mathrm{~Hz}, 26 \mathrm{~Hz}$, etc.

Measurements of the magnetic components of the Schumann resonances at the fundamental frequency are $10^{-12}$ Tesla. The typical magnetic field strength for the oscillations within the cerebral cortices from the scalp associated with thinking are $10^{-12} \mathrm{~T}$ (Persinger, 2014). The electric field component of the Schumann resonance is about $1 \mathrm{mV} \cdot \mathrm{m}^{-1}$; an almost identical potential difference is measured for the human brain. The primary 
sources of the Schumann resonance are lightning strikes over the entire surface of the earth (but primarily in the equatorial regions). They occur about $44 \pm 5$ times per second, or $44 \pm 5 \mathrm{~Hz}$ (Christian et al., 2003). The primary model for human consciousness is electroencephalographic activity around $40 \mathrm{~Hz}$. The current density within a lightning discharge is about $10^{5} \mathrm{~A}$ per $\mathrm{m}^{2}$. The equivalent current distribution for an action potential as it moves along the axon barrel, if it were scaled to a meter, would be $10^{5} \mathrm{~A}_{\text {per }} \mathrm{m}^{2}$ (Persinger, 2012b).

Recent measurements by Saroka and Persinger (2014) indicated that several of the power fluctuations within the lower harmonics of the Schumann resonances are coupled in real time with electroencephalographic power of human brains. The concurrence is about 150 to $300 \mathrm{msec}$, which is equivalent to about 2 or 3 microstates of consciousness (Koenig et al., 2002) on average about once every $30 \mathrm{~s}$. This duration is the classic interval of short-term memory. Consequently the conditions that could promote the convergence of evolutionary properties that contribute to consciousness within human brains exist and would be consistent with Teilhard de Chardin's predictions. There are many themes that "run through evolution". The first three involve "groping profusion, constructive ingenuity and indifference towards whatever is not future and totality". "There is also a fourth heading which embraces all of them: global unity.

\section{Temporal Continuity of Consciousness: "Immortality"}

Teilhard de Chardin's essential units imply a type of immortality or a temporal duration that approaches that of the age of the universe. There is quantitative support for this potential based upon the specific quantity or intensity of the magnetic field strength found within the human brain during cognition which is similar to that displayed by the Schumann Resonance generated by the earth. This potential is seen by calculating the induced magnetic moment upon an electron which corresponds to a change in angular velocity (the motion of a particle in a circle around a central point). An electron moving in a closed path produces a magnetic field. The induced magnetic moment $(\Delta m)$ is:

$$
\Delta m=-\left[e^{2} r^{2} / 4 m_{e}\right] B
$$

where " $e$ " is the fundamental unit charge of $1.6 \times 10^{-19} \mathrm{~A} \cdot \mathrm{s}, r=$ the radius of the Bohr atom $\left(5.1 \times 10^{-11} \mathrm{~m}\right), m_{\mathrm{e}}$ is the mass of an electron $\left(9.1 \times 10^{-31} \mathrm{~kg}\right)$ and $B$ is the strength of the applied magnetic field.

The solution for this aggregate when applied within the magnetic field strength $\left(10^{-12} \mathrm{~T}\right)$ associated with the $40 \mathrm{~Hz}$ pattern of consciousness or of the earth-ionospheric resonance within which all human beings are exposed, becomes $10^{-40} \mathrm{~A} \cdot \mathrm{m}^{2}$ (the unit for magnetic moment) which is the same as Joules per Tesla. To obtain only energy, this ratio must be multiplied again by another magnetic field strength. If either the Schumann intensity is applied, or the average intensity of the intragalactic magnetic fields (Neronov \& Vovk, 2010) is utilized, the energy is $10^{-52} \mathrm{~J}$.

This value becomes a potential central point for Teilhard de Chardin's premise for two reasons. First, if the photon, the particle that has been attributed with "timelessness" and may be a source of intercalation between the energies of space and time, is at rest its mass would be about $10^{-52} \mathrm{~kg}$ (Tu et al., 2005). In other words of $\mathrm{m}^{2} \cdot \mathrm{s}^{-2}$ approached unit, $10^{-52} \mathrm{~kg}$ would be convergent with $10^{-52} \mathrm{~J}$. Secondly, if this energy of $10^{-52} \mathrm{~J}$ is divided into the reference value for quantum solutions, Planck's constant $\left(6.626 \times 10^{-34} \mathrm{~J} \cdot \mathrm{s}\right)$, the solution is time (seconds). The actually value is about $4 \times 10^{17} \mathrm{~s}$ which is effectively the age of the universe. Although this does not necessarily prove the immortality of the self, it does suggest a temporal continuance for the information associated with the energy that produced the change.

Applying the "Omega" concept, in its ultimate principle, can only be a "distinct Centre radiating at the core of a systems of centres; a grouping in which personalization of the All and personalization of the elements reach their maximum, simultaneously, without merging, under the influence of supremely autonomous focus of union" (p. 289), the quantification indicates that a representation of the "whole" superimposed upon the individual unit (the electron's angular motion) without necessarily merging, reflects a central radiating core. The core could be the rest mass-equivalent energy of a photon whose temporal value is the age of the universe.

\section{Expansion of the Noosphere (The Sphere of Human Thought)}

Under the section of "The Ultimate" the definition of the noosphere is expanded and articulated more succinctly. He states "we have as yet no idea of the possible magnitude of 'noospheric' effects. We are confronted with human vibrations resounding by the million-a whole layer of consciousness exerting simultaneous pressure 
upon the future and the collected and hoarded produce of a million years of thought” (p. 313). Continuing, "Under the increasing tension of the mind on the surface of the globe, we may begin by asking seriously whether life will not perhaps one day succeed in ingeniously forcing the bars of its earthly prison by getting into psychical touch with other focal points of consciousness across the abysses of space...the Universal and Personal...grow in the same direction and culminate simultaneously in each other" (p. 285). "Consciousness would thus finally construct itself by a synthesis of planetary units". If we substitute terms such as "psychical" for "cognitive" and "consciousness" for "electromagnetic or energetic patterns", the expanding noosphere develops physical characteristics. This physical basis of consciousness is not unique. A mathematical form with specific biochemical substrates, the microtubules, has been thoroughly developed by Hammeroff and Penrose (2014).

In previous quantifications, Persinger (2008) showed that the amount of energy contained within the space occupied by the earth's magnetic field was sufficient to store the energies from all of the action potentials and hence potentially all of the thoughts of every person that has ever lived. The assumption of his model was that during the labile duration, of about $30 \mathrm{~min}$, for the electrical stage of memory before it is represented as newly formed spines (newly synthesized proteins) on dendrites of neurons, there is also the representation of this information in electromagnetic form within the space occupied by the earth's magnetic field through the spherical (earth-ionosphere) waveguide, i.e., the Schumann Resonances.

The physical parameters for the earth's magnetic field as well as the conductivity between the ionosphere and the earth's surface solve for this specific duration. In addition recent measurements by Professor Kevin Saroka showed brief (150 to $300 \mathrm{~ms}$ ) coherence between Schuman frequency powers recorded a continent away (Italy) as well as locally (Sudbury, Ontario) within the EEG patterns for dozens of human participants. The coherence occurred once every approximately $30 \mathrm{~s}$. This is the first physical evidence that electromagnetic mediated information could be shared between the brain and the entire surface of the earth's shell within which Schumann resonances occur.

Assuming that the functional energy from neurons associated with percepts and thoughts is about $10^{-9} \mathrm{~J}$ per second and that each person lives for about 2 Gigaseconds (70 years), the total "pure" energy from simply the individual's lifetime of "cognition" would be about 1 Joule. Even with conservative estimates of 100 billion human beings over the history of the planet, only about $10^{11}$ Joules would have been represented within that space. In comparison the energy associated with the earth's entire magnetic field is within the order of $10^{18} \mathrm{~J}$. Stated alternatively, all of the energy from every thought of every person who has ever existed would only occupy about one-ten billionth of the total potential.

Teilhard de Chardin's concept of expansion of a "human universal consciousness" once a critical threshold had been achieved was prescient. The electric field between the ionosphere and the earth surface within which the Schumann resonances are generated and all humans are immersed is about $10^{-12} \mathrm{~A}$ (amps) per $\mathrm{m}^{2}$. According to Volland (1982) the total mean on flat ground from these currents is about 2 pA per square meter. The difference between the surface over the oceans or lands is negligible.

Assuming the average of $2 \times 10^{-6} \mathrm{~V}$ (2 microVolts) for the primary $40 \mathrm{~Hz}$ activity through the cerebral cortical tissue associated with cognition and consciousness for which the intracellular resistivity is $\sim 2 \Omega \cdot \mathrm{m}$, the current would be $10^{-6} \mathrm{~A} \cdot \mathrm{m}^{-1}$ and when applied across the averaged linear distance of the cerebrum $\left(\sim 10^{-1} \mathrm{~m}\right)$, would be $10^{-7} \mathrm{~A}$ per human brain. For $7 \times 10^{9}$ human brains, the summed current is $7 \times 10^{2} \mathrm{~A}$ and when spread over the surface of the earth $\left(5 \cdot 10^{14} \mathrm{~m}^{2}\right)$ is about $1.4 \cdot 10^{-12} \mathrm{~A} \cdot \mathrm{m}^{-2}$. For comparison the mean of the air-earth current over land is about $(2.0 \pm 0.3) \times 10^{-12} \mathrm{~A} \cdot \mathrm{m}^{-2}$ (Volland, 1982).

Hence the current density, on average, for human beings in the process of thinking or consciousness over the surface of the planet is beginning to approach the current density of the air-earth current over land. When the values converge which could occur with the addition of only another 3 billion people or a total of 10 billion people, the current density of the earth and the human species of a whole would enter a condition where "...the Universal and Personal...grow in the same direction and culminate simultaneously in each other" (p. 285). Whether or not this is the threshold at which "Under the increasing tension of the mind on the surface of the globe, we may begin by asking seriously whether life will not perhaps one day succeed in ingeniously forcing the bars of its earthly prison" remains to be discerned.

Obviously there are clusters of places where the current density would be less or more. This may not be important. Teilhard de Chardin's premise would require the human species to behave as a unit or a whole. Although each person would contribute their quantification to the whole, an integrator would be required. One of the most likely candidates is the earth's magnetic field within which almost every living system is immersed. 
The geomagnetic field displays the conditions that would satisfy the noosphere that encircles the earth through evolution of consciousness and complexity. Both the geomagnetic field and the Schumann Resonance have been present since the first amino acids were formed by the lightning discharges through ammonia, hydrogen, oxygen, methane and carbon dioxide (Graf \& Cole, 1974).

The physical form for connectivity in space-time is magnetic diffusivity ( $\mathrm{m}^{2}$ per $\mathrm{s}$ ). The process is defined as the ratio of conductivity of a medium divided the magnetic permeability of a vacuum. For physiological saline within each human brain the conductivity $(\sigma)$ is $2.1 \mathrm{~S} \cdot \mathrm{m}^{-1}$ (Siemens per meter). When divided by magnetic permeability $(\mu)$ which is $1.26 \times 10^{-6} \mathrm{~N} \cdot \mathrm{A}^{-2}$ (Newtons per square A), the result is a diffusivity term of $1.3 \times 10^{6} \mathrm{~m}^{2}$ per s. Assuming 7 billion human cerebrums each with a cross-sectional surface area of $10^{-2} \mathrm{~m}^{2}$, the total surface area of "cerebral surface" connected by the magnetic flux lines of the earth would be $7 \times 10^{7} \mathrm{~m}^{2}$ would be about one minute. Even if the conductivity varied by several units or the cross-sectional area of cerebrums were slightly larger the potential passive connectivity between any one cerebrum and all other cerebrums would be within the time of less than 10 min (Persinger, 2013c). Such passive connectiveness increases the degrees of freedom within the whole aggregate for increasing complexity.

There is also a dynamic component. The product of magnetic flux density measured in Tesla $\left(\mathrm{kg} \cdot \mathrm{A}^{-1} \cdot \mathrm{s}^{-2}\right)$, current density $\left(\mathrm{A} \cdot \mathrm{m}^{-2}\right)$ and magnetic diffusivity $\left(\mathrm{m}^{2} \cdot \mathrm{s}^{-1}\right)$ is $\mathrm{kg} \cdot \mathrm{s}^{-3}$ or $\mathrm{W} \cdot \mathrm{m}^{-2}$. There is one potential process by which brains that share the earth's magnetic field or common current densities might be associated. Assuming the value of $10^{-12} \mathrm{~T}$ for either the functional magnetic field intensity for human cerebral activity or the first harmonic of the Schumann Resonance, $10^{-12} \mathrm{~A} \cdot \mathrm{m}^{-2}$ current density for either the earth-air value or the average for all of the brains of the human population, and $10^{6} \mathrm{~m}^{2} \cdot \mathrm{s}^{-1}$ (from $(\mu \cdot \rho)^{-1}$ where $\mu$ is magnetic permeability $(4 \pi$ $\times 10^{-7} \mathrm{~N} \cdot \mathrm{A}^{-2}$ ) and $\rho=$ the inverse of $2 \cdot \Omega \cdot \mathrm{m}$ or $0.5 \mathrm{~S}$, the irradiant flux density would be $\sim 10^{-18} \mathrm{~W} \cdot \mathrm{m}^{-2}$.

The significance of this relatively small flux density becomes apparent when it is applied over the cross-sectional area of human cerebrum (cortex), about $10^{-2} \mathrm{~m}^{2}$ (Pakkenberg \& Gundersen, 1997). The resulting value is $10^{-20} \mathrm{~W}$ or $\mathrm{J} \cdot \mathrm{s}^{-1}$. This is the unit energy for a single action potential. Energy is also the product of the potential difference (voltage) and unit charge. For the typical voltage associated with the $40 \mathrm{~Hz}$ pattern correlated with consciousness, which is $2 \mu \mathrm{V}$, the resulting energy would be $3.2 \times 10^{-25} \mathrm{~J}$. When divided by Planck's constant the frequency is within $\pi$ of the neutral hydrogen wavelength. This could be the wavelength (connecting the force per Planck's voxel to be $10^{-20} \mathrm{~J}$ ) that would allow distributions "across the abysses of space".

\section{Excess Correlation and Non-Locality}

The presumption of locality requires an intricate serial sequence of events that are sufficiently adjacent in space-time for forces to be influential or for a mediating process to affect directly the adjacent events. As a result there is a discrete latency associated with this propagation that can be measured and predicted. This intrinsic process is the basis of cause-and-effect.

However, the concept of non-locality challenges this assumption. For example, the philosophy of Ernst Mach assumes the prominence of the Universe. From this context any one part of the universe is affected by all of the other parts. For it to be valid an integrating process would be required that includes the entire set (the universe) and occurs more or less instantaneously. There is evidence for brief temporal connections across universal boundaries derived from the fundamental constants (Persinger \& Koren, 2013). The presence of non-locality is inferred by the persistence of "excess correlation" between two events at distances that cannot be accommodated by the latencies expected for "locality" and ordinary mechanisms of cause and effect or the properties of the medium (such as the velocity of light) that moderate the effect.

Dotta et al. (2011) demonstrated the "proof in principle" of this concept experimentally by exposing the cerebrums of pairs of volunteers to an identical sequence of magnetic field patterns that were rotated counterclockwise in the horizontal plane at the level of the ears. The circular array of point magnetic fields consisted of 8 separate solenoids each separated by 45 degrees that was placed around the head like a laurel. Each member of the pair was separated by about $10 \mathrm{~m}$ and sat in comfortable chairs within separate, closed rooms. The fields were generated from this circularly array of eight solenoids where the rate of change of the presence of the field in each of the solenoids (as the field pulses were rotated around the heads) was controlled by computer software. From our perspective this experimental configuration produced the "same" temporal configuration within in two different loci.

Dotta et al. (2011) based the conceptual operations of the circularly, changing angular velocities of magnetic 
fields around the head from the usual behavior of photons when their rest masses were assumed to be non-zero. Tu et al. (2005) had summarized the physical theory and mathematical derivation of these different properties. One of these characteristics is that group velocities (the speed of the whole packet of photons) become different from their phase velocities (movement of photons within the packet). Dotta and his colleagues rotated the fields around the heads at different rates than the repetition frequencies of the patterns contained within the field. They found that when one of the yoked pair was exposed to bright light in one room there was increased emission of photons from the right side of the brain of the other member of the yoked pair, separated by 10 meters, and sitting in another room that was hyperdark.

The effect only occurred if there were two stages to the exposure and if the magnetic fields were present. The first involved a circularly rotating magnetic field with an accelerating group velocity associated with a diminishing phase velocity for a few minutes followed by a decelerating group velocity but an increasing phase velocity for about twice that time. No other combinations were effective. A constant velocity of rotation also was not effective. In subsequent studies Dotta and Persinger (2012) found that when two chemical luminescent reactions both shared the same optimal field simultaneous injection of the reactant in both locations doubled the photon emissions. During this specific sharing of fields parameters the two loci behaved as if they were superimposed within the same space. This effect was demonstrated as far as $3 \mathrm{~km}$, which was the furthest distance tested.

Slightly different equipment indicated that complex brain activity could be coordinated when the two subjects were exposed to the same configuration of magnetic fields (a changing angularity velocity around the head was still required) and separated by as far as $300 \mathrm{~km}$ (Burke et al., 2013). This “excess correlation” of reactions between two locations when the two spaces share the circularly rotating magnetic field with different rates of angular velocity has been found for the most essential chemical reactions associated with brain function, such as shifts in pH or the relative acid-base levels (Dotta et al., 2013).

Recently Karbowski et al. (2015) showed that pairs of dishes containing melanoma cells sharing similar rotating magnetic fields exhibited excess correlation over a distance of approximately $2 \mathrm{~km}$. When one dish in the pair was injected with hydrogen peroxide that diminished cell growth by the following day the cells in the other dish, $2 \mathrm{~km}$ away, that had received no injections showed a proportional diminishment of growth. These results indicate that under the appropriate conditions that would imbue photons with additional properties superposition of different loci behave as the same space. This would produce an environment where the noosphere might be maintained.

\section{Twenty-First Century Technology and the Noosphere}

One of the conditions that facilitate the emergence of a complex whole that is greater than the sum of its parts or elements is the presence of a force or energy within which all of the elements are immersed. For example a parallel bundle of a million wires moving through magnetic flux lines or the field moving across the bundle generates a configuration of a changing field (a "tensor") that may reflect the characteristics of the elements. The whole field would also display properties that add to these characteristics as secondary representations. During the evolution of the geosphere and biosphere these immersive forces and energies included the geomagnetic field and the Schumann Resonances.

The biosphere, particularly from the contributions of the human species, has generated additional immersive forces. They include the communication systems defined by relatively restricted $\mathrm{GHz}$ bands that are remarkably adjacent to the $1.42 \mathrm{GHz}$ signature that defines the neutral hydrogen line that itself permeates the universe. Although the wavelengths for the hydrogen line and for $\mathrm{GHz}$ bands associated with modern communication systems occur within the range of the circumference and diameter of the human cerebrum, the more fundamental effect would occur from beats.

Beats are the differences between frequencies. In the simplest form if a $1000 \mathrm{~Hz}$ and $1010 \mathrm{~Hz}$ tone were presented simultaneously, the experience would also include the subtraction of the two: a $10 \mathrm{~Hz}$ "tone". It would be an emergent property and from some perspectives a "virtual” condition derived from the derivate of the change. The differentiating individual features for cell phone communications primarily depend upon the sub-frequencies or lower frequencies and patterns superimposed upon the carrier. There will be some point in the near future where the density of communication requirements are such that the subtractions will result in near-continuous "beat" patterns that overlap with the range of brain activity, that is, between 1 and $100 \mathrm{~Hz}$. 
Although the "energy" levels from global communications systems have been argued to be "too weak" to be cerebrally effective, calculations suggest otherwise. For example if the radiant flux density or power density of a $\mathrm{GHz}$ signals was $10 \mathrm{~mW}$ per square meter, which is a common intensity within a few meters of kitchen microwave when it is operational, the energy available to just the soma or cell body of a neuron with a cross sectional area of $10^{-10} \mathrm{~m}^{2}$ would be $10^{-12}$ Joules per second (or Watts). This is the same order of magnitude as the amount of energy supplied from glucose metabolism within the cell to maintain its organelles and functions.

Within the last decade the emergence of the World Wide Web has allowed "information" in its electromagnetic form to be present in space-time in a manner never previously possible. This information is accessed visually and through earbud devices, worn more and more frequently by members of the population, which allows direct proximity to the associated magnetic fields within the listener's brain space. The outer regions (cortices) of the temporal lobes are only about $2 \mathrm{~cm}$ away from the spaces in which the earbud devices are placed. We have measured magnetic field intensities in the range of 0.1 to 1 microTesla from these electronic devices (Saroka \& Persinger, 2011). The magnetic energy within the volume of the cerebral cortices would be sufficient to affect, potentially at least, millions of neurons.

There is another immersive energy that Teilhard de Chardin may have considered the "precipitant" to produce the conditions to allow the expansion of consciousness into non-local space. This additional energy is that associated with the direct interfacing between computer electronics and human brains. The most productive and expanding forms of this synergism are the escalating numbers of virtual and real-time engagement softwares or "games" that couple the brain with complex electronics. Many of these conditions produce "the flow state". It has been defined by Csikszentmihalyi (1990) as a feeling complete and energized focus of activity associated with a high level of enjoyment and fulfillment.

Our laboratory (Lavallee \& Persinger, 2010) specializes in relating the quantitative changes in brain activity during typical cognitive states and those associated with "mystical" or "spiritual" perceptions. The array of sensors placed over the scalp according to conventional neurological procedures is effectively an aggregate of small volt meters. The amplitudes of the potential differences between each sensor that fluctuate between about 1 and $40 \mathrm{~Hz}$, although fast transients around $400 \mathrm{~Hz}$ can be measured, are about $2 \mu \mathrm{V}$ to $20 \mu \mathrm{V}$ per Hz. These "ripples" originate from structural arrangement of the billions of cells in the cerebral cortices and are superimposed upon the "steady potential" or "d.c." potential for the whole brain which is between 10 and $30 \mathrm{mV}$ (about 1000 times stronger). With appropriate software we can map the transient spatial patterns of voltage changes during discrete increments of time and construct three-dimensional images of the current sources (with resolutions of about 1 $\mathrm{cm}$ ) any where within the cerebral volume.

Direct measurements in our laboratory of the quantitative electroencephalographic activity of people engaging in the virtual perceptions generated by computer technology indicate there are frequent but intermittent occurrences of a "flow state". This is a specific pattern of activity within portions of the brains that could allow access to the Schumann domains and the interfacing with other brains engaging in similar activities. This flow state involves the right hemisphere, which is the region associated with "mystical" experiences (Persinger et al., 2010), creativity, and the feeling of expansiveness in space and time. It is also the region, particularly when the right parahippocampal gyrus is involved, that is likely to mediate the coherence between the cerebral activity of human beings on this planet and the activity within the earth's spherical wave guide within which the Schumann Resonances intercalate. In our experiments more detailed analyses indicated that the right occipital, temporooccipital junction, parietal region and orbitofrontal cortices are particularly activated during the flow state (see Figure 1).

The flow state was maximal when there was a decrease of activation in the left inferior frontal region but increased power within the $7-8 \mathrm{~Hz}, 27-28 \mathrm{~Hz}$, and $42 \mathrm{~Hz}$ bands within the left temporal regions. Activation of this area is correlated with experiences of the self in general and the re-representation of memory specifically. We interpret this pattern as the flow state being a "state of consciousness" where there is a diminished personal intention (the "release" of sense of self to another) and planning in conjunction with more reliance upon experiencing passively the information within the left hemisphere as it is transformed from right hemispheric processes. If large numbers of people on the planet engaged in the technology that produced this configuration within the cerebrums and the specific subjective experiences, the conditions would be met potentially for the creation of a noosphere or the sphere of human thought with specific physical properties, as predicted by Teilhard de Chardin. 

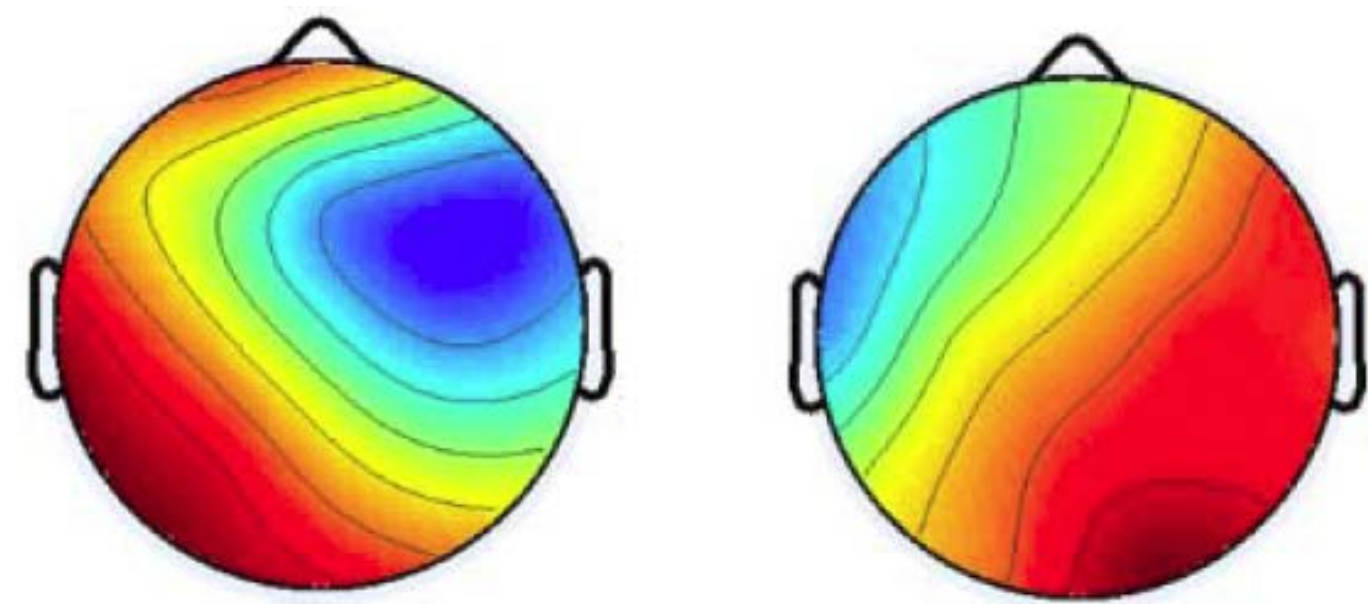

Figure 1. An example of the power shift within critical frequencies as measured by quantitative electroencephalography that would allow intercalation with an external field consistent with "the noosphere" that occurs during the "flow state" of interaction with virtual experiences in computer programs. Red indicates maximum power; blue indicates minimum power. The map on the left indicates the activation of the left caudal temporal region while the map on the right indicates the right occipitotemporal region. The condition would be consistent with the "confluence" between the electromagnetic cognitive states of the individual and the physical correlate (Schumann Resonance immersed in the earth's magnetic field) of Teilhard de Chardin's noosphere.

\section{Conclusion}

Three of Teilhard de Chardin's concepts: the prominence of "spiritual energy", the emergence of a sphere of human thinking (the noosphere) and the expansion of consciousness beyond the terrestrial domain can be supported by quantification of known parameters of the human cerebral cortices and the fundamental energies and forces that permeate the universe. There is evidence that what he called "spiritual energies" may be quantifiable to values similar in magnitude to those that operate within quantum levels. There are sufficient physical electromagnetic and temporal similarities between the Schumann Resonances of the earth-ionospheric cavity and the cerebrums of the human population to allow the conditions that could create the "noosphere". The persistence of alternations in magnetic moment by changes in angular velocity of the simplest unit, the electron-proton pairing, by superimposing the magnetic field strength associated with thinking upon the background intergalactic magnetic field, could qualify for the physical foundation required for immortality and the convergence at the Omega Point.

\section{Conflicts of Interest}

The authors declare no conflict of interest

\section{Acknowledgements}

Thanks to Dr. Blake T. Dotta for his technical skills. Special thanks to Professor Ghislaine F. Lafreniere and the Jesuits from the Department of Philosophy and Religious Studies who inspired discourse at the University of Sudbury during the late 1960s and early 1970s for introducing us Teilhard de Chardin. This article is dedicated to his memory and to his courage.

\section{References}

Bohr, N. (1958). Atomic Physics and Human Knowledge. New York: Wiley and Sons.

Burke, R. C., Gauthier, M. Y., Rouleau, N., \& Persinger, M. A. (2013). Experimental Demonstration of Potential Entanglement of Brain Activity Over $300 \mathrm{Km}$ for Pairs of Subjects Sharing the Same Circular Rotating, Angular Accelerating Magnetic Fields: Verification by s_LORETA, QEEG Measurements. Journal of Consciousness Exploration \& Research, 4, $35-44$. 
Christian, H. J., Blakeslee, R. J., Boccippino, D. J., Boeck, W. L., Buechler, D. E., Driscoll, K. T. et al. (2003). Global Frequency and Distribution of Lightning as Observed From Space by the Optical Transient Detector. Journal of Geophysical Research, 108, ACL 4-1-ACL 4-15. http://dx.doi.org/10.1029/2002JD002347

Chung, S. J. (2015). On the Possible Deeper Structure of Leptons and Quarks: A View of “Ultron”-“Logotron” Theory. Open Journal of Philosophy, 5, 302-314. http://dx.doi.org/10.4236/ojpp.2015.55038

Csikszentmihalyi, M. (1990). Flow: The Psychology of Optimal Experiences. New York: Harper \& Row.

Dotta, B. T., Buckner, C. A., Lafrenie, R. M., \& Persinger, M. A. (2011). Photon Emissions from Human Brain and Cell Culture Exposed to Distally Rotating Magnetic Fields Shared by Separate Light-Stimulated Brains and Cells. Brain Research, 1388, 77-88. http://dx.doi.org/10.1016/j.brainres.2011.03.001

Dotta, B. T., Murugan, N. J., Karbowski, L. M., \& Persinger, M. A. (2013). Excessive Correlated Shifts in pH with Distal Solutions Sharing Phase-Uncoupled Angular Accelerating Magnetic Fields: Macro-Entanglement and Information Transfer. International Journal of Physical Sciences, 8, 1783-1787.

Dotta, B. T., \& Persinger, M. A. (2011). Increased Photon Emissions From the Right But Not the Left Hemisphere While Imagining White Light in the Dark: The Potential Connection Between Consciousness and Cerebral Light. Journal of Consciousness Exploration and Research, 2, 1463-1473.

Dotta, B. T., \& Persinger, M. A. (2012). Doubling of Local Photon Emissions When Two Simultaneous, Spatially Separated, Chemiluminescent Reactions Share the Same Magnetic Field Configurations. Journal of Biophysical Chemistry, 3, 72-80. http://dx.doi.org/10.4236/jbpc.2012.31009

Dotta, B. T., Saroka, K. S., \& Persinger, M. A. (2012). Increased Photon Emission from the Head While Imagining White Light in the Dark is Correlated with Changes in Electroencephalographic Power: Support for Bokkon's Biophoton Hypothesis. Neuroscience Letters, 513, 151-154. http://dx.doi.org/10.1016/j.neulet.2012.02.021

Eddington, A. (1981). Nature of the Physical World. Ann Arbor, MI: University Press.

Fels, D. (2009). Cellular Communication through Light. PLoS ONE, 4, 35086. http://dx.doi.org/10.1371/annotation/8d99ccc5-cc76-44f4-b468-d63e42e0b9e1

Graf, F. E., \& Cole, E. R. (1974). Precambrian ELF and Abiogenesis. In M. A. Persinger (Ed.), ELF and VLF Electromagnetic Field Effects (pp. 243-273). New York: Plenum Press.

Gurwitch, A. G. (1926). Das Problem der Zelltilung Physiologish Betrachtet. Berlin: Springer. http://dx.doi.org/10.1007/978-3-642-90660-2

Hammeroff, S., \& Penrose, R. (2014). Consciousness in the Universe: A Review of the "Orch OR” Theory. Physics of Life Reviews, 11, 39-78. http://dx.doi.org/10.1016/j.plrev.2013.08.002

Houwelling, A. R., \& Brecht, M. (2008). Behavioural Report of a Single Neuron Stimulation in Somatosensory Cortex. Nature, 451, 65-69. http://dx.doi.org/10.1038/nature06447

Karbowski, L. M., Murugan, N. J., \& Persinger, M. A. (2015). Experimentally-Induced Inhibition of Growth in Melanoma Cell Cultures Separated by Two km When Both Share Excess Correlation Magnetic Fields: Macroscopic Evidence for Free-Space Quantum Teleportation? Journal of Signal and Information Processing, 6, 39-48. http://dx.doi.org/10.4236/jsip.2015.61004

Koenig, T., Prichep, L., Lehmann, D., Sosa, D. V., Braker, E., Kleinlogel, H., Ishehart, H., \& John, E. R. (2002). Millisecond by Millisecond, Year by Year: Normative EEG Microstates and Developmental Stages. NeuroImage, 16, 41-48. http://dx.doi.org/10.1006/nimg.2002.1070

Lavallee, C. F., \& Persinger, M. A. (2010). A LORETA Study of Mental Time Travel: Similar and Distinct Electrophysiological Correlates of Re-Experiencing Past Events and Pre-Experiencing Future Events. Consciousness and Cognition, 19, 1037-1044. http://dx.doi.org/10.1016/j.concog.2010.06.008

Li, C.-Y. T., Poo, M.-M., \& Dan, Y. (2009). Burst Spiking of a Single Cortical Neuron Modifies Global Brain State. Science, 324, 643-646. http://dx.doi.org/10.1126/science.1169957

Llinas, R., \& Pare, D. (1991). Of Dreams and Wakefulness. Neuroscience, 44, 521-525. http://dx.doi.org/10.1016/0306-4522(91)90075-Y

Merz, J. T. (1965). A History of European Thought in the Nineteenth Century. New York: Dover.

Neronov, A., \& Vovk, I. (2010). Evidence for Strong Extragalactic Magnetic Fields from Fermi Observations of TeV Blazars. Science, 328, 73-76. http://dx.doi.org/10.1126/science.1184192

Nickolaenko, A., \& Hayakawa, M. (2014). Schumann Resonance for Tyros: Essentials of Global Electromagnetic Resonance in the Earth-Ionosphere Cavity. New York: Springer. http://dx.doi.org/10.1007/978-4-431-54358-9

Nordlund, T. (2015). The Physics of Augustine: The Matter of Time, Change and an Unchanging God. Religions, 6, $221-224$. http://dx.doi.org/10.3390/rel6010221 
Pakkenberg, B., \& Gundersen, H. J. G. (1997). Neocortical Neuron Numbers in Humans: Effects of Sex and Age. Journal of Comparative Neurology, 384, 312-320. http://dx.doi.org/10.1002/(SICI)1096-9861(19970728)384:2<312::AID-CNE10>3.0.CO;2-K

Persinger, M. A. (2008). On the Possible Representation of the Electromagnetic Equivalents of All Human Memory within the Earth’s Magnetic Field: Implications for Theoretical Biology. Theoretical Biological Insights, 1, 3-11.

Persinger, M. A. (2010). 10 $0^{-20}$ Joules as a Neuromolecular Quantum in Medicinal Chemistry: An Alternative Approach to Myriad Molecular Pathways. Current Medicinal Chemistry, 17, 3094-3098. http://dx.doi.org/10.2174/092986710791959701

Persinger, M. A. (2012a). Brain Electromagnetic Activity and Lightning: Potentially Congruent Scale-Invariant Quantitative Properties. Frontiers in Integrative Neuroscience, 6, 19. http://dx.doi.org/10.3389/fnint.2012.00019

Persinger, M. A. (2012b). Potential Origins of a Quantitative Equivalence between Gravity and Light. The Open Astronomy Journal, 5, 41-43. http://dx.doi.org/10.2174/1874381101205010041

Persinger, M. A. (2013a). Experimental Evidence That Hubble’s Parameter Could Be Reflected in Local Physical and Chemical Reactions: Support for Mach's Principle of Imminence of the Universe. International Letters of Chemistry, Physics and Astronomy, 11, 86-92. http://dx.doi.org/10.18052/www.scipress.com/ilcpa.16.86

Persinger, M. A. (2013b). A Possible Explanation for the Vacuum Catastrophe. International Journal of Astronomy and Astrophysics, 4, 171-180.

Persinger, M. A. (2013c). Billions of Human Brains Immersed Within a Shared Geomagnetic Field: Quantitative Solutions and Implications for Future Adaptations. The Open Biology Journal, 6, 8-13.

http://dx.doi.org/10.2174/1874196701306010008

Persinger, M. A. (2014). Schumann Resonance Frequencies Found Within Quantitative Electroencephalographic Activity: Implications for Earth-Brain Interactions. International Letters of Chemistry, Physics and Astronomy, 11, 24-32. http://dx.doi.org/10.18052/www.scipress.com/ILCPA.30.24

Persinger, M. A., \& Koren, S. A. (2013). Dimensional Analyses of Geometric Products and Boundary Conditions of the Universe: Implications for a Quantitative Value for the Latency to Display Entanglement. The Open Journal of Astronomy, 6, 10-13. http://dx.doi.org/10.2174/1874381101306010010

Persinger, M. A., Koren, S. A., \& Lafreniere, G. F. (2008). A Neuroquantological Approach to How Much Thought Might Affect the Universe. NeuroQuantology, 6, 262-271. http://dx.doi.org/10.14704/nq.2008.6.3.182

Persinger, M. A., Saroka, K. S., Koren, S. A., \& St-Pierre, L. S. (2010). The Electromagnetic Induction of Mystical and Altered States within the Laboratory. Journal of Consciousness Exploration \& Research, 1, 808-830.

Popp, F. A. (1979). Coherent Photon Storage of Biological Systems. In F. A. Popp, H. Koenig and W. Prechka (Eds.), Electromagnetic Bio-Information (pp. 123-149). Munich-Wein-Baltimore: Urban and Schwarzenberg.

Puthoff, H. L. (1989). Gravity as a Zero-Point-Fluctuation Force. Physical Review A, 39, 2333-2342. http://dx.doi.org/10.1103/PhysRevA.39.2333

Rose, D. (2006). Consciousness: Philosophical, Psychological and Neural Theories. Oxford: Oxford Press.

Saroka, K. S., \& Persinger, M. A. (2011). Detection of Electromagnetic Equivalents of the Emotional Characteristics of Words: Implications for the Electronic-Listening Generation. Open Behavioral Sciences Journal, 5, 24-27. http://dx.doi.org/10.2174/1874230001105010024

Saroka, K. S., \& Persinger, M. A. (2014). Quantitative Evidence for Direct Effects between Earth-Ionosphere Schumann Resonances and Human Cerebral Cortical Activity. International Letters of Chemistry, Physics and Astronomy, 39, 166-194. http://dx.doi.org/10.18052/www.scipress.com/ILCPA.39.166

Teilhard de Chardin, P. (1959). The Phenomenon of Man. New York: Harpers.

Trushin, M. V. (2009). Light Mediated Conversation Among Microorganisms. Microbiological Research, 159, 1-10. http://dx.doi.org/10.1016/j.micres.2003.11.001

Tu, L. C., Luo, J., \& Gilles, G. T. (2005). The Mass of the Photon. Progress in Physics, 68, 77-130. http://dx.doi.org/10.1088/0034-4885/68/1/R02

Volland, H. (1982). Handbook of Atmospherics (Vol. 1). Boca Raton, FL: CRC Press. 